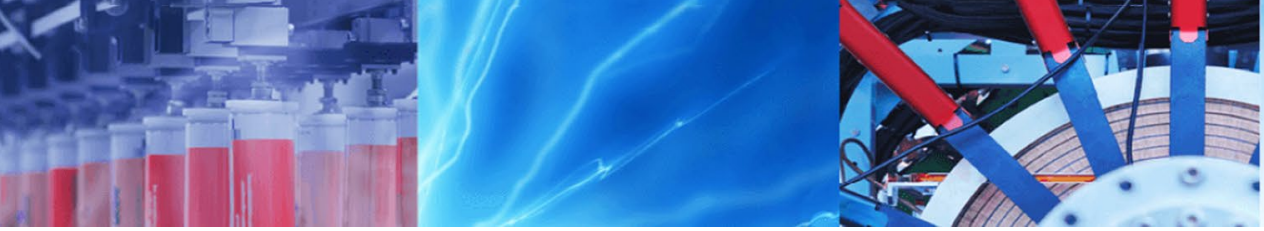

Research Article

\title{
Design and simulation of a control algorithm for peak-load shaving using vehicle to grid technology
}

\author{
Juan Daniel Arango Castellanos ${ }^{1} \cdot$ Harish Dhanasekaran Velayutha Rajan ${ }^{2} \cdot$ Ann-Kathrin Rohde ${ }^{1} \cdot$ Dimitri Denhof $^{1}$. \\ Michael Freitag ${ }^{1,3}$
}

(c) Springer Nature Switzerland AG 2019

\begin{abstract}
The continuous growth of renewable energy sources and combined heat-power generation plants in public infrastructures allow them a certain degree of independence from the public power grid. Nevertheless, during the day, the power demand fluctuates, creating peak loads that cannot be fulfilled by the generated power and must be covered by the public power grid. Peak loads could be diminished by saving energy produced during low power demand periods and returning it during the high demand periods. The use of electric vehicles is expected to keep increasing in the next years and the use of vehicle-to-grid technology allows electric and hybrid cars to return stored energy to the power grid. An algorithm to evaluate the vehicle-to-grid technology as a solution for peak reduction, also called peak shaving, for public infrastructure was developed. The purpose of the algorithm is to predict the optimal charging and discharging schedule of the battery from electric vehicles in a parking space so that the peak load demands are met without sacrificing the driving demands of the electric vehicle (EV) users. This takes into consideration factors like the state of charge of the batteries and the mobility needs of the vehicle user. The algorithm takes the driving demands and mobility needs as constraints, and schedules the charging and discharging of the EVs during their stay in a parking place in order to reduce the peak load demands by supplying stored electrical power from EV batteries back to the grid. In order to validate the algorithm, three test case scenarios representing different parking patterns were generated using random and statistical distributed parameters. It is concluded that vehicle-to-grid technology can be used to reduce peak demands, where the number of EVs and their stay hours in a parking place represent the most critical parameters for the effectivity of the peak reduction. This implies that in the future, public infrastructure could profit better from renewable energy sources by adding loading stations for EVs that are vehicle-to-grid capable.
\end{abstract}

Keywords Vehicle-to-grid (V2G) $\cdot$ Electric vehicles (EVs) $\cdot$ Peak demand $\cdot$ Charging $\cdot$ Discharging $\cdot$ Peak shaving

\section{Background}

As a part of energy conservation, the installation and use of renewable energy power generation plants or the Combined Heat-Power (CHP) generation plants are enormously increasing [1]. Depending on the size of the CHP and subvention politics of the country it can be economical to meet the entire electric power demand by using the above mentioned self-sufficient energy sources exclusively [2]. In this kind of public infrastructures, the decentralized power generation plants supply an increasing share of energy. Decentralized energy plants reduce energy losses due to transportation and distribution; nevertheless, these decentralized plants mostly work together with external power supplies to guarantee that the peak load demands can be covered. Peak loads are generated due

$\triangle$ Juan Daniel Arango Castellanos, ara@biba.uni-bremen.de | ${ }^{1}$ BIBA - Bremer Institut für Produktion und Logistik GmbH, Hochschulring 20, 28359 Bremen, Germany. ${ }^{2}$ Faculty of Physics and Electrical Engineering, University of Bremen, Otto-Hahn-Allee 1, 28359 Bremen, Germany. ${ }^{3}$ Faculty of Production Engineering, University of Bremen, Badgasteiner Str. 1, 28359 Bremen, Germany.

SN Applied Sciences (2019) 1:951 | https://doi.org/10.1007/s42452-019-0999-x 
to high-energy demands within short time slots. Such loads could be partially or totally covered by decentralized energy plants if the additional energy that is produced by the power plant while the power demand is low would be stored, for example at night, and used during the high power demand periods. In general, batteries are used to store electric energy, but this resource is not always available sufficiently.

The use of Electric Vehicles (EVs) batteries used in transportation could represent a solution for this problem. The number of Plug-In Hybrid Electric Vehicle (PHEVs) and Battery-Electric Vehicles (BEVs) increases daily as the increasing concern of environmental aspects such as greenhouse gas emissions encourages the use of EVs. Moreover, politic decisions toward EVs can increase the amount of these cars, for example, the German federal government has set its target of increasing the number of registered electric vehicles to 1 million by the year 2020 [3]. According to statistical data, most of the working days, these electric vehicles are parked for almost $20 \mathrm{~h}$ a day [4]. The batteries of EVs that are parked for a long time in public infrastructures could be used as energy storage devices with the help of Vehicle to Grid (V2G) technology and therefore be used to reduce the peak load demand fluctuations. A bidirectional charging unit would allow the power to flow from power grid to the battery during charging and also back from the battery to the power grid during discharging [5]. However, a control algorithm is needed for scheduling the charging and discharging of the electric vehicle batteries. With a proper scheduling of the charging and discharging cycles, the peak load demands fluctuations could be reduced. A concept to meet the peak load demand fluctuations by using the batteries from EVs is proposed in this paper.

\section{Theoretical background}

\subsection{Power generation}

Renewable energy generation enables the industries and commercial buildings to harvest energy from wind, solar or biogas and to be more self-sufficient in meeting their power demand. They try to reduce the dependency on the external power supply and to produce their own power to manage their electricity demand [6]. However, shifting to renewable, self-generated energy involves various challenges as renewable energy is not available consistently. It rather depends on the environmental aspects such as weather conditions for wind and solar energy.

\subsection{Peak load demand}

In power systems, there exists an imbalance in the amount of demand and supply. Unplanned power plant outages, non-disposable loads, load forecast errors or climatic conditions supplementary lead to high peak demands in the system [7]. Satisfying high peak demands is costly for the concerned power plant in terms of efficiency and reliability. This also has an economical repercussion, for example, for the German market, the peak load prices were at an average of 7 Euro/MWh higher than baseload price for a period over 7 days in December based on the data collected from EPEXSpot auction data [8, 9].

The uncertain energy feed-in of renewable sources additionally affects the power system efficiency and affects the demand-supply balance in the system. With the increase of distributed energy generation that integrates renewable sources of hybrid consumer and supplier, there exists a keen need to manage the peak demand with the available resources in the system. Energy storage devices could be an economical way of solving this problem of overcoming peak demands in the decentralized power generation system considering the vast development in battery technology [10]. This will make the decentralized power plants to run more self-sufficient, reducing their dependency on the external power supplies. However, for a small sized decentralized power plant in a commercial infrastructure, installing and maintaining large capacity energy storage devices is expensive.

\subsection{Electric vehicles}

There has been a huge shift in the transportation sector in the recent years. Electric Vehicles (EVs) are a pollution free, efficient transportation system as compared to conventional vehicles. Over the past years, because of the improvements in battery technology and the rise in oil prices, the EVs are found to be economically advantageous in short-distance commuting [11]. The number of EVs is increasing steadily, in Germany a vast rise of the number from EVs was registered [12]. Many countries provide a lot of subsidies and privileges to EV owners as e.g. Germany, where EVs are exempted from annual vehicle tax for 5 years [11]. In the European market, Norway has the largest share of EVs where $29 \%$ of the vehicles are EVs [13].

\subsection{Charging stations}

Along with the increase of EVs, there also exists an increase in the need for public electric vehicle charging stations (EVCSs). The batteries of the EVs (BEVs and PHEVs) are 
charged by connecting them to the charging stations with a plug-in cable. Germany has more than 14,000 public charging points and Europe provides more than 92,000. These EVCSs are mostly designed as parking spaces with charging option and are supported with a software identification system that holds information about the vehicles and the owner's account details. This information is then used for payments and the power delivery [14]. Charging stations have to manage the power transfer between the grid and the EVs. Uncontrolled charging, i.e. charging at a time point of arrival or at the time point the user wants to charge his EV irrespective of the grid conditions affects the grid efficiency [15]. Integration of these charging units to the grid involves a good planning so that these charging units do not congest the grid traffic or deteriorate the grid efficiency. A coordinated charging system is necessary in order to avoid adding peak load demand into the grid because of EVs charging [16].

\subsection{Vehicle to grid technology}

Vehicle to Grid (V2G) is as the name says, the ability to transfer electrical power from an electric vehicle to the electric grid [17]. This technology uses the excess of battery power of electric vehicles for grid services such as frequency regulation and peak demand supply management. V2G is achieved with a bidirectional power converter available in the charging station that could convert the power from $A C$ to $D C$ and also from DC to AC [4]. When EVCSs are connected with the grid and could facilitate bidirectional power supply, EVs could be used as mobile energy storage devices. It can save the power in their batteries by charging during the less demand period or the valley period and it can supply the power back to the grid by discharging when the peak demand occurs.

Several incentive-based schemes have been developed to encourage EV users to participate in grid services. For example, an EV user can charge the car at night when the electricity prices are low and discharge it at high electricity prices during the morning peak periods. A simulationbased analysis of the German power market until 2030 showed that the V2G scheme is more advantageous in improving the capacity factors in decentralized small and mid-sized power plants [18]. When V2G is considered, there are various areas that still need to be explored to analyze its full potential for grid services and their economic advantage. In this paper, the major focus is analyzing the results of scheduled charging and discharging of electric vehicles based on the load demand and driving demand and to analyze its effect on the load demand profile in various situations. V2G charge and discharge algorithm were designed for implementing V2G for peak shaving applications without compromising the driving demands. This algorithm requires the forecasted load profile of the infrastructure and the EV parking plan of the parking space.

\section{Methods}

\subsection{Load demand profile}

The load profile used for the test scenarios follows a typical load profile in a commercial infrastructure, as shown in Fig. 1. A peak threshold value is taken in order to determine the peak load hours in the load profile. The peak threshold is either the rated capacity of power production plant or the maximum of the base load consumption which is used by the infrastructures. A valley threshold is also taken to determine the valley hours, which is a period when the power consumption is less than the base load. Thus, the examined load profile shows peak power consumption during the morning hours (10:00-13:00 h) and in

Fig. 1 Sample load profile [19]

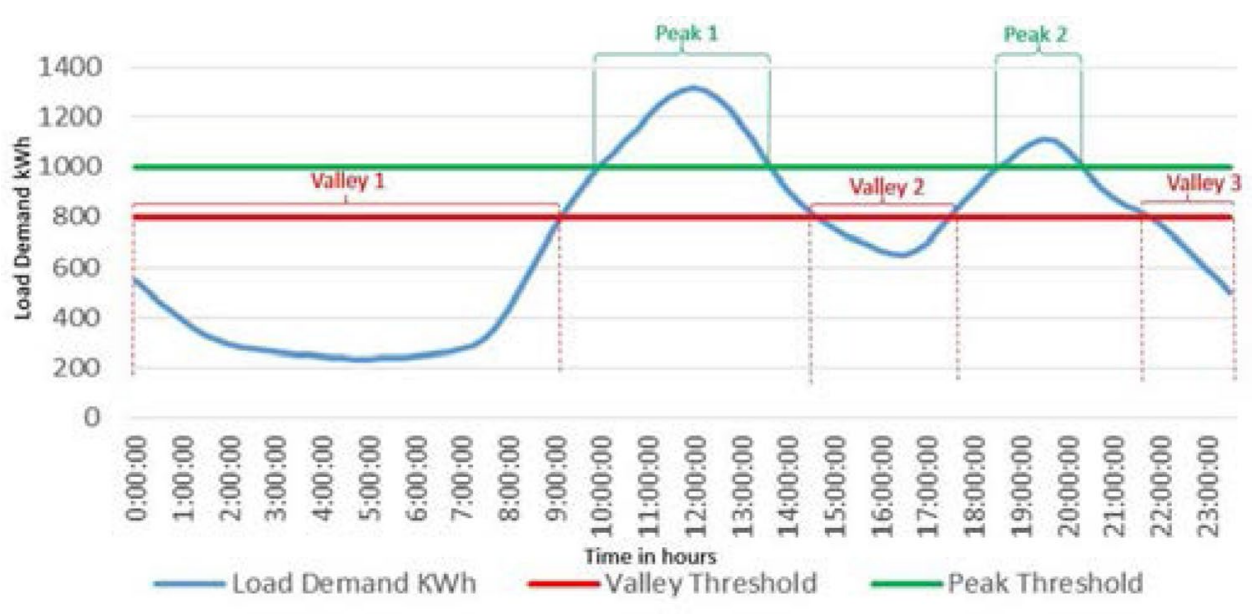


Table 1 Basic description of most common EVs in Germany

\begin{tabular}{llll}
\hline Brand & Model & Battery $(\mathrm{kW} \mathrm{h})$ & Range (Miles) \\
\hline Renault & Zoe & 17.6 & 100 \\
Kia & Soul & 30 & $130-190$ \\
VW & Golf & 35.8 & $150-200$ \\
BMW & i3 & 33 & $130-160$ \\
Tesla & Model S & 70 & $230-240$ \\
\hline
\end{tabular}

the evening (18:00-20:00 $\mathrm{h}$ ) and very low power consumption during the night hours (00:00-05:00 h).

\subsection{EVs and charging station}

Based on the registrations between January 2017 and November 2017, the basic description of the most popular battery electric vehicles running in Germany is mentioned in Table 1 [20].

The listed vehicles have an average battery capacity of $37.3 \mathrm{kWh}$. The definition of the test fleet of electric vehicles for the test cases is based on the specifications of the vehicles, mentioned in Table 1. It is further assumed that these vehicles support $\mathrm{V} 2 \mathrm{G}$ technology.

The charging station considered for this research is base on the existing charging station from RWE, which has the specifications listed below [21]:

- Manufacturer: RWE

- Product type: RWE Mobility eStation Smart Device

- No. of Charging points: 2

- Output Power: 22 kW, 400 V AC, 3 Phase, 32A.

\subsection{EV parking plan}

Several EV parking plans are modeled to represent the various parking scenarios. The scenarios are classified according to the parking pattern of the EV owners. Each scenario varies fundamentally in the period of stay in the parking space. MATLAB was used to model and evaluate each scenario. The three scenarios generated in the simulation are named as Short-term scenario, Modified Short-term scenario, and Long-Term scenario. Table 2 shortly describes the parameters used in the scenarios. The scenarios have been developed taking into account the fact that the possible infrastructure is an airport, where different parking situations can occur. Each scenario is extended on the basis of three different cases involving a different number of electric vehicles such as 50, 100 and 500 vehicles. This provides the opportunity to analyze the changes in the percentage of peak reduction with an increase in the number of electric vehicles. By comparing the three scenarios with the three different cases that include a different
Table 2 Parameters used in each scenario

\begin{tabular}{|c|c|}
\hline Parameter & Description \\
\hline Number of EV & 50,100 and 500 for each scenario \\
\hline BMS & Battery management system of the EV \\
\hline SOC & State of charge from the EV \\
\hline Battery capacity (kWh) & $\begin{array}{l}\text { Randomly assigned storage capacity } \\
\text { depending on the car models from } \\
\text { Table } 1\end{array}$ \\
\hline Length of stay & $\begin{array}{l}\text { Assigned parking period of stay for each } \\
\text { EV }\end{array}$ \\
\hline Parking period & $\begin{array}{l}\text { The time during EV are allowed to park. It } \\
\text { can be also understood as the simula- } \\
\text { tion time }\end{array}$ \\
\hline
\end{tabular}

number of EVs, the significance of $\mathrm{V} 2 \mathrm{G}$ can be visualized for different scale of EVs. The results could be used to support further research on real-time implementation of $V 2 G$ technology. The three scenarios will be analyzed using the V2G charge-discharge algorithm. The efficiency of peak demand reduction is also compared for all three scenarios.

The electric vehicle's battery management system (BMS) provides information about the remaining battery state of charge $(\mathrm{SOC})$ and the remaining available driving range. The participation in the $\mathrm{V} 2 \mathrm{G}$ services requires the consent of the Electric vehicle owner. For the considered scenarios the permission of the owner is always assumed as granted.

The EV parking plan in each scenario includes the considered EVs on the parking space and their respective parameters. These parameters include the vehicle's specifications such as model, type, and rated battery capacity. The battery information, which includes SOC of the battery at the entry and exit, is generated based on the conditions for each scenario. The parking information such as the arrival time, departure time and the stay hours are also generated based on the conditions for each scenario. The parameters to be generated are determined using the statistical distribution functions. Statistical distributive functions are used to generate the EV parking plan in order to comply with the conditions and assumptions considered for each scenario. By using statistical distribution functions instead of fixed values, the scenarios can represent real scenarios where the parking plan is different for each EV. Table 3 compares the main parameters for each scenario.

\subsection{Short-term scenario}

The Short-Term parking scenario implies the parking pattern over a period of $24 \mathrm{~h}$. It represents the parking patterns on parking lots of office buildings or employees' parking lots at commercial infrastructures. The employees usually have a regular work schedule over 
Table 3 Overview of the scenarios

\begin{tabular}{llll}
\hline & Short-term scenario & Modified short-term scenario & Long-term scenario \\
\hline Possible scenario & Office building & Commercial building & Airport \\
Parking period & $24 \mathrm{~h}$ & $24 \mathrm{~h}$ & 8 days \\
SOC on entry & $40-60 \%$ & $30-70 \%$ & $10-90 \%$ \\
Length of stay & $5-10 \mathrm{~h}$ & $1-12 \mathrm{~h}$ & $3-8$ days \\
Minimal SOC at exit & $50 \%$ & $50 \%$ & $80 \%$ \\
Arrival time & 7 a.m. until 10 a.m. & Anytime & Anytime \\
\hline
\end{tabular}

the weekdays and the electric vehicles are used for commute to work and back home.

A research study shows that EVs are charged mostly at private charging points (household chargers). This leads to the hypothesis that the EVs are charged during the night and used for commuting to work during the day. It is also better to charge the EVs at home at night as the power demand in the grid is lower. The average distance traveled by an EV is approximately $40 \mathrm{~km}$. According to the average battery capacity (about $40 \mathrm{kWh}$ ) of the vehicles considered in this analysis and taking into account the consumption rate of $0.2 \mathrm{kWh} /$ $\mathrm{km}$, the average driving distance traveled in 1 day could easily be covered with $50 \%$ of the battery capacity [22, 23].

Therefore, the modeling of this scenario assumes that the SOC at the entry (on arrival) to the parking place is on average $50 \%$. The average length of stay is $8 \mathrm{~h}$. Considering that the return trip after working time is similar to the distance traveled in the morning, the EV needs 50\% SOC to commute back home. The EVs must be charged at least $50 \%$ at the end of their stay to ensure that the driving requirements are met. Thus, the algorithm is constrained to schedule the EVs charging and discharging in such a way that the SOC at the exit (at departure time) is at least $50 \%$.

This scenario is generated with three different populations of EVs such as a fleet of 50,100 and 500 vehicles. The arrival time is distributed randomly between 7:00 and 10:00 a.m. The staying hours are generated using a normal distribution function with an average value of $8 \mathrm{~h}$ and a standard deviation of $1 \mathrm{~h}$, as shown in Fig. 2 . This scenario would represent the real-time scenario in which not all vehicles depart or arrive at the same time. The normal distribution function generates the values with the required randomness within the defined range. Similarly, the SOC at the entry point is assigned using the normal distribution function with an average value of $50 \%$ and a standard deviation of $5 \%$, so that not all EVs have exactly $50 \%$, but rather a SOC value between 40 and $60 \%$. In this way, the parameters for all vehicles are assigned in this scenario.

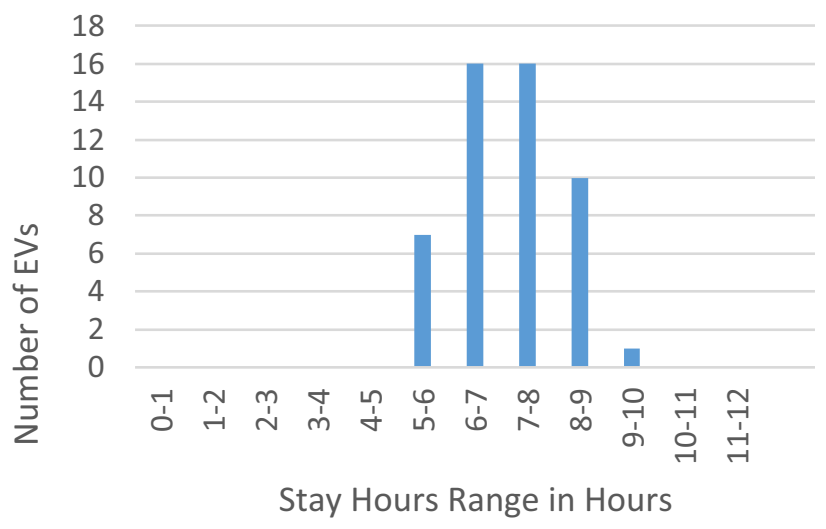

Fig. 2 Stay hours distribution for 50 EVs-Short-term scenario

\subsection{Modified short-term scenario}

The modified Short-term parking scenario implies the parking pattern over a period of $24 \mathrm{~h}$. It represents parking lots of public commercial buildings or public parking lots. This scenario comprises EVs with different parking hours. The EVs arrive randomly throughout the day and their staying hours are randomly distributed and vary from at least 1-12 h. The stay hours are not evenly distributed as in Short-term parking. This way, the normal public parking scenarios such as at a shopping mall or visitor parking scenarios could be covered in the EV parking plan.

Considering the dynamics of the scenario, the SOC of the EV'S at the entry is distributed over a wide range (30-70\%). By extending the range, the EVs have a more dynamic range of values for $\mathrm{SOC}$ at the entry. In this scenario, the SOC at the exit is again set to at least $50 \%$. The EVs must be charged at least until $50 \%$ at the end of their stay period to ensure that the driving requirements are met. During the parking periods, the load demand profile of the infrastructure might consist of peak and valley periods. The EVs will be scheduled to charge during valley slots to ensure the $\mathrm{SOC}$ at the exit is $50 \%$. If the EV has more than $50 \%$ SOC at the entry, it could discharge during the peak period until the SOC reaches $50 \%$. In this scenario, not all the vehicles can be discharged due to various reasons such as stay period could be short, or because there 


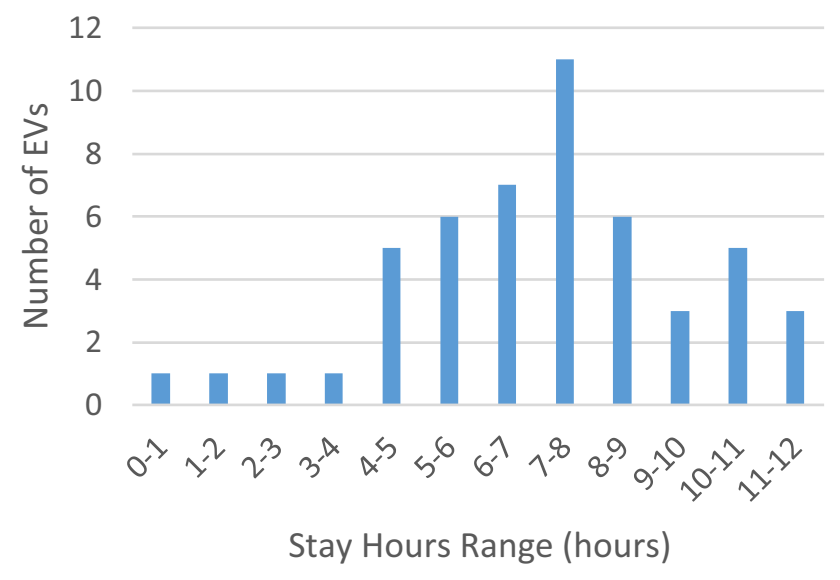

Fig. 3 Stay hours distribution for 50 EVs—-modified short-term

is no peak load period existing between the stay periods or because they do not have enough power in their batteries to be discharged.

This scenario is also generated with three different populations of EVs such as a fleet of 50, 100 and 500 vehicles. The arrival time is distributed randomly over the $24 \mathrm{~h}$ of a day, using random time points between the specific times. Similar to the Short-term scenario, the staying hours and the SOC for all the EVs are assigned using statistical distribution functions such as normal distribution function. The stay hours are created using a normal distribution function with a mean of $8 \mathrm{~h}$ and a standard deviation of $3 \mathrm{~h}$, as shown in Fig. 3. This would represent a realistic scenario, where not all vehicles depart or arrive at the same time. Additionally, each vehicle has a specific length of stay in the parking that varies randomly from 1 to $12 \mathrm{~h}$. Similarly, the SOC at the entry point is assigned for all electric vehicles with the help of normal distribution function with a mean value of $50 \%$ and a standard deviation of $10 \%$. This ensures that the EVs SOC at entry varies from 30 to $70 \%$.

\subsection{Long-term scenario}

The long-term parking scenario implies the parking pattern over a period of 10 days. It represents the parking patterns in parking places such as in airports, railway stations or public parking houses. The EVs parked here can be used as energy storage devices to support the power grid. EVs are considered to be arriving and departing at the parking place randomly all through the day.

In modeling the scenario, it is assumed that the $\mathrm{SOC}$ at the entry is randomly determined between 10 and $90 \%$. For all vehicles, the SOC at the exit is to be between 80 and $90 \%$. The Long-term parking scenario is usually observed at airports or railway stations. The distance people travel to the airport is generally greater than the distance they travel every day between the office and home. Therefore, the energy demand for the return journey after the parking period might be higher than that of the short-term and modified short-term scenario. Moreover, the planning of the trip a week in advance is uncertain, so that at the end of the parking period, the EVs would be charged until the SOC reaches $80-90 \%$ to meet the driving requirements of the user. In this scenario, mostly all vehicles are charged at night time, when the load demand is normally low and discharged in the morning during peak load demand periods.

Similar to the other two scenarios, this scenario is also generated with three different populations of EVs such as a fleet of 50, 100 and 500 vehicles. The arrival time is distributed randomly throughout the $24 \mathrm{~h}$ of a day. The stay hours and the SOC for all the EVs are assigned for all the EVs using a statistical distribution functions such as cumulative normal distribution. The stay hours are created using a normal distribution function with a mean of $150 \mathrm{~h}$ ( 6 days) and a standard deviation of $20 \mathrm{~h}$, as shown in Fig. 4. This would represent a realistic scenario, where not all vehicles depart or arrive at the same time and it ensures that the stay hours are varying randomly from 3 to 8 days. Similarly, the SOC at the entry point is assigned for all electric vehicles with the help of normal distribution function with a mean value of $50 \%$ and a standard deviation of $10 \%$.

\subsection{Constraints and limitations}

For this research, a few constraints and assumptions are defined. The real hardware entities, required for V2G, are only available in the developmental phase. Additionally, research on the advantages and disadvantages of the V2G technology found out, that one of the major drawbacks of $\mathrm{V} 2 \mathrm{G}$ is the degradation of the vehicle's battery life [15, $18,24]$. Therefore, in order to avoid the degradation of the

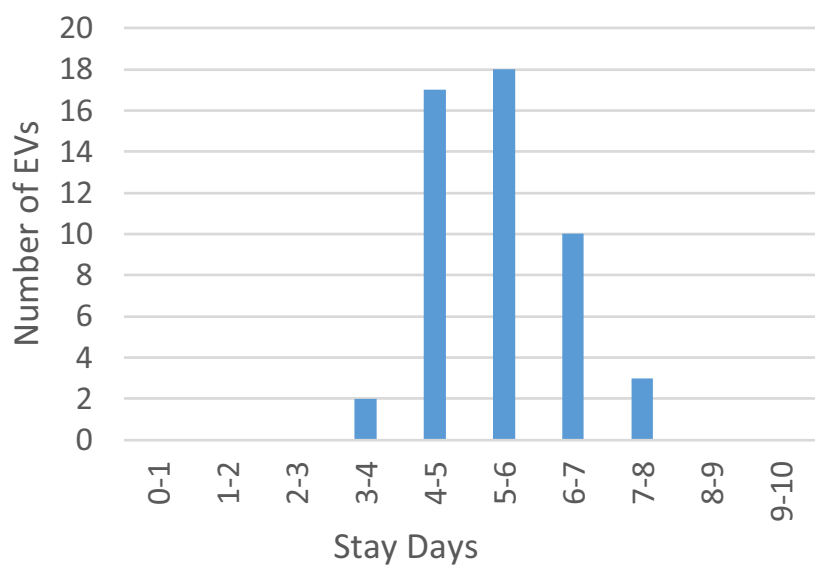

Fig. 4 Stay days distribution for 50 EVs-long-term 
battery life of the vehicles and to analyze the potential of V2G implementation for different scenarios, some constraints were defined.

This paper majorly lands on the assumption that there will be a large number of electric vehicles in use in the future and that sufficient charging stations will be installed in public parking places. It is also assumed that the charging stations are able to provide bidirectional support for transporting power from the grid to the car and also back from the car to the grid in accordance with the CHAdeMO protocol [23].

The following conditions and assumptions were considered in this study:

- It is not assumed that the EVs batteries are charged completely to $100 \%$ or discharged totally to $0 \%$ SOC. Charging a battery to $100 \%$ or discharging a battery to $0 \%$ would greatly affect the battery performance over the years. The Depth of Discharge (DOD) is the opposite of the State of Charge (SOC). SOC represents the percentage of battery capacity remaining in the battery. DOD is the percentage of battery capacity remaining in the battery. DOD is an important factor in characterizing the deterioration of battery life. A research study lists out the major factors that cause battery degradation and the results show that completely draining the battery accelerates the battery life degradation [24]. Therefore, the simulations underlying this contribution assume that the $\mathrm{SOC}$ will be maintained between 10 and $90 \%$.

- The lifetime of the EV battery decreases after a finite number of charge and discharge cycles. V2G will increase the charging and discharging cycles as its batteries are used to maintain driving demand in addition to fundamental functionality [15, 24]. Therefore, a compromise has to be made when EVs are used in V2G applications. In order to minimize the charge-discharge cycles, the algorithm defines a restriction when scheduling the charging of the battery. The scheduling of the charging process is limited in such a way that the EV reaches $90 \%$ SOC and the discharging are scheduled until the EV reaches $10 \%$ SOC. This is to avoid frequent charging and discharging which in turn increases the charge and discharge cycles. Therefore, it is taken as a constraint in this algorithm stating that the EVs are scheduled to be charged when the SOC reaches less than $10 \%$ and they are scheduled to be charged until the SOC reaches $90 \%$. Similarly, they are only discharged if the SOC is equal to or greater than $90 \%$ and they are discharged until the SOC reaches $10 \%$.

- The charging and discharging rate are assumed to be linear and equal, based on the EV charging station power capacity to simplify the analysis. The amount of energy exchanged between the vehicle battery and the charging station is the same and considered to be equal to the capacity of the charging station. In this paper, the EVs can charge and discharge at a rate of $22 \mathrm{~kW}$ per hour according to the specifications of the RWE charging stations, which corresponds to $5.5 \mathrm{~kW}$ in $15 \mathrm{~min}$ time slot [21]. Additionally, the charging-discharging and the grid losses are neglected to simplify the analysis made by the algorithm.

- This research focuses mainly on designing an algorithm and analyzing the potential of V2G implementation. The implementation of the algorithm in the real scenario requires an interactive information system design that acts as a communication interface between the V2G system and EV user and also between the energy system and V2G system. Such an information system design goes beyond the scope of this research.

\subsection{Algorithm description}

The algorithm defines the periods consisting of time slots of $15 \mathrm{~min}$, since the considered load profile has load requirements for every $15 \mathrm{~min}$. The peak slots and valley slots are determined from the load profile as well as from the peak and valley threshold. The algorithm creates a charge-discharge time window for each EV from the EV parking plan. The EVs are only eligible to charge and discharge their batteries within this time window. This ensures that the driving requirements are not compromised, i.e. even after participating in V2G, the EV has enough valley slots to charge until the battery reaches the required SOC before their departure. As soon as the time window for charge and discharge is fixed, the EVs are scheduled to charge and discharge at the available peak and valley periods respectively. The algorithm decides to either charge or discharge based on the SOC of their batteries. If the EV has a high SOC, it will be scheduled to be discharged at the next available peak period. If it has a low SOC, it will be scheduled to be charged in the next available valley period. The charging is scheduled during the valley period in such a way that the charging takes place during the time window when the load demand reaches the minimum. The discharging is scheduled during the peak period in such a way that the discharging takes place during the time window when the load demand reaches the maximum. Once the EVs are scheduled for either charging or discharging, the time window for discharging and charging is reduced to the next available period within their parking period. The scheduling for the next peak and valley slots is then repeated until there is no peak or valley period within the charge-discharge window. After that, the EV is finally charged in the last valley slot to ensure the driving demands are met. Finally, the EVs are charged 
during the low demand slots, and if they have sufficient power, they can discharge power back into the grid during high demand periods.

The V2G charge-discharge algorithm is explained in the flowchart in Fig. 5. It shows the flow of the algorithm step by step. Once the parking plan and SOC for each vehicle has been generated and imported, the peak and valley slots during their stay period are calculated. Then, based on the scenario conditions and the conditions of the EV's driving demands, the charge-discharge window is determined. Once the charge-discharge window is determined, the EVs are scheduled to charging or discharging based on the power available in the respective valley and peak slots. Once scheduling is complete, the charge-discharge window is reduced to the next available slots and the scheduling is further carried out. The scheduling is repeated until the charge-discharge window ceases to exist or if there are no more peak and valley slots in the EV parking period.
Fig. 5 V2G charge-discharge algorithm flow chart

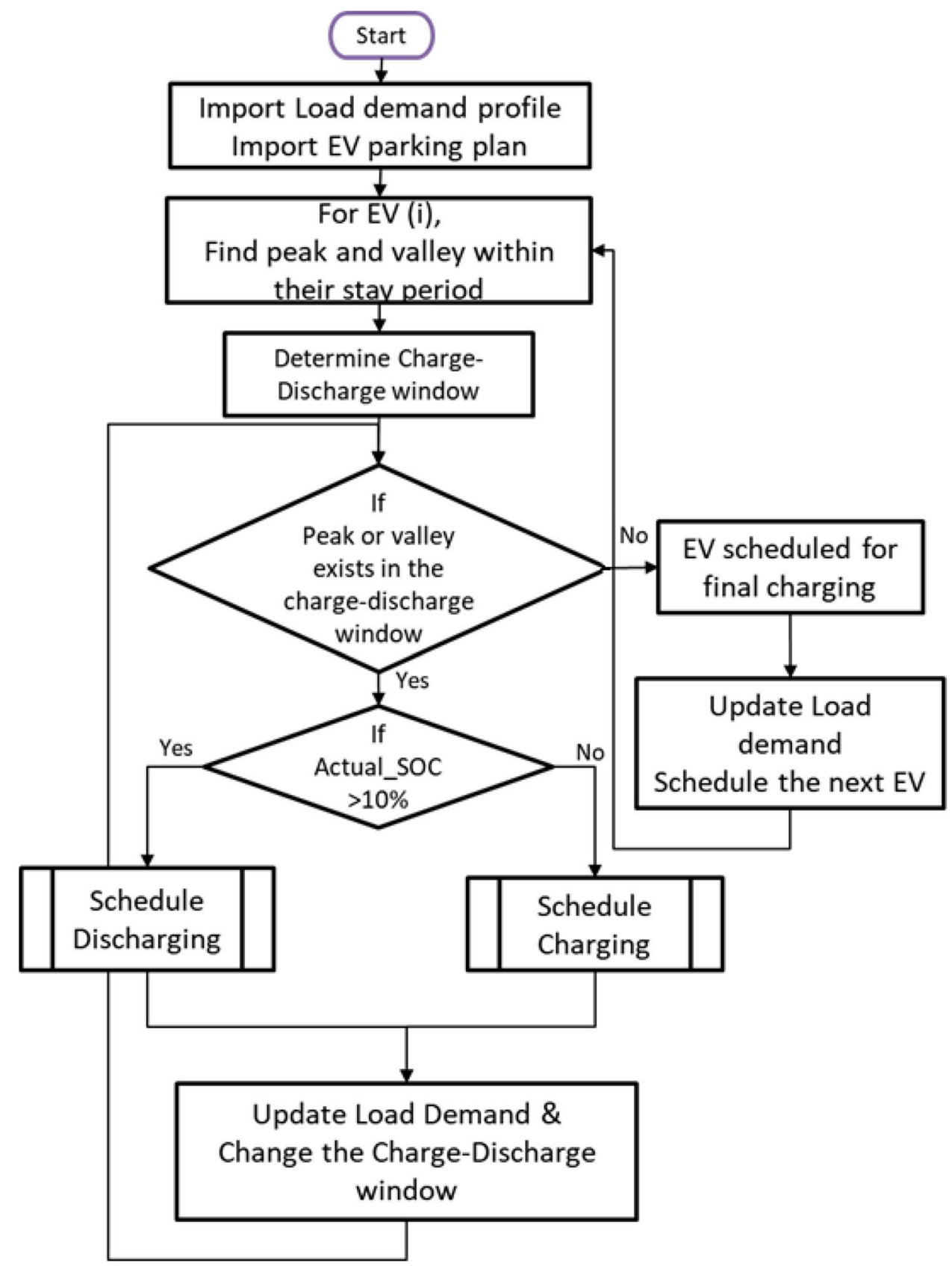




\section{Results and discussion}

The V2G charge-discharge algorithm is applied in the simulation of three scenarios of EVs park plan, each with three different cases of EVs populations. This algorithm determines the charging and discharging schedule of the EVs during their parking period. Each vehicle is assigned its respective time slots to be charged and/or discharged. The load demand profile is changed on the basis of this EV charging and discharging schedule. The load demand is increased during the EV's charging slots and reduced during EV's discharging slots. The load demands before and after V2G scheduling are evaluated with two evaluation factors, such as percentage of peak reduction and load factor. These factors determine the efficiency of peak reduction using V2G in each scenario.

The percentage of peak reduction is a quantitative measure of the total reduction of the peak demand value in a load profile. It is the percentage decrease of the peak demand in the load profile before and after scheduling, as shown in Eq. 1. It can also be explained as the ratio of the amount of reduction of the peak value after scheduling to the amount of the peak demand value before scheduling. This is calculated using Eq. (1).

PeakRed. $=\left(\frac{\sum\left(\text { Peak_demand }_{\text {before }}-\text { Peak_demand }_{\text {after }}\right)}{\sum \text { Peak_demand }_{\text {before }}}\right) * 100$

where Peak_demand ${ }_{\text {before }}$ is the total amount of peak demand existing in the load profile before scheduling, and Peak_demand ${ }_{\text {after }}$ is the total amount of peak demand existing after scheduling the EVs charging and discharging.

In general, the load factor is defined as the ratio between the average load demand and the maximum demand over a period of time. In load profile analysis, the load factor is a measure of the variation in power generation and consumption [25]. If the load factor is high, this means that the load profile has a low peak demand and the energy consumption is approximately constant. If the load factor is low, then the load profile has a high peak demand and the energy consumption is not constant. The load factor is calculated for the load profile before and after applying the V2G scheduling algorithm. The difference of the load factor determines how effectively the peak is reduced and to what extent a balance between demand and supply is achieved in all scenarios. Load Factor before $_{\text {and Load Factor }}$ after denotes the load factor of the load profile before and after scheduling respectively and they are calculated using Eqs. (2) and (3).

Load Factor $_{\text {before }}=\left(\frac{\text { Average OldLoadDemand }}{\text { Maximum Peak Demand }}\right)$
Load Factor ${ }_{\text {after }}=\left(\frac{\text { Average newLoadDemand }}{\text { Maximum Peak Demand }}\right)$

The three scenarios are tested with three different cases that vary in the number of EVs. The evaluation factors: PeakRed., Load Factor before $_{\text {and Load Factor }}$ after are calculated in each scenario. Considering the variations, due to random assignment of the EV parameters in the EV parking plan, the results of the algorithm need to be generalized to make it reliable. Therefore, the algorithm is repeated for 10 simulation trials in each test case. This means that 10 different $\mathrm{EV}$ parking plans are created in each test scenario and in each case. The V2G charge-discharge algorithm is then applied to each trial and the resulting load profiles are evaluated using the above evaluation factors. The average value of the evaluation factors for the 10 trials is calculated as the final result.

\subsection{Comparison}

The results show that the percentage of peak reduction in the Long-term parking scenarios is generally higher compared to Short-term and Modified Short-term parking scenario, mostly because the stay hours of the EV include both the peak hours and a sufficient number of valley hours. The EVs can charge their batteries during the valley hours and are available for discharge during peak demand hours. In this way the EVs are scheduled to be charged to their full power during the low demand hours at night time and the stored energy is made available for coping with the peak hours during the day. In Short-term and Modified Short-term parking, the peak reduction is lower compared to Long-term parking, because the EV's stay hours are less and they need to be charged back to a minimum of $50 \%$ SOC before departure to meet the driving demand of EV owners. The EVs have fewer time slots to charge and discharge in these scenarios as their stay is shorter. As a result, the percentage of peak reduction is lower in the Short-term and Modified Short-term parking scenario than in the Long-term parking scenario. Table 4 shows the value of the percentage of peak reduction in all scenarios and the comparison chart is shown in Fig. 6.

Table 4 Average peak reduction-comparison results

\begin{tabular}{llll}
\hline Case & \multicolumn{2}{l}{ Average peak reduction $(\mathrm{kWh})$} \\
\cline { 2 - 4 } & Short-term & $\begin{array}{l}\text { Modified Short- } \\
\text { term }\end{array}$ & Long-term \\
\hline Case 50 & 19.04 & 14.92 & 51.08 \\
Case 100 & 35.52 & 28.33 & 74.52 \\
Case 500 & 80.96 & 79.68 & 100 \\
\hline
\end{tabular}


Similarly, comparing the load factor in all scenarios, the increase in the load factor is higher in Short-term and Long-term scenarios than in the Modified Shortterm scenario. This indicates that V2G scheduling is more effective when applying peak shaving in an infrastructure where there is a fixed schedule for EV parking times. The increase in load factor values in all scenarios not only demonstrates the peak reduction, but also proves that V2G contributes to a better balance between demand and supply in the load profile. The load factors in all scenarios are shown in Table 5 and the comparison chart in Fig. 7.
Fig. 6 Peak reduction in 3 scenarios

Table 5 Load factor comparison over scenarios

Fig. 7 Average load factor in 3 cases

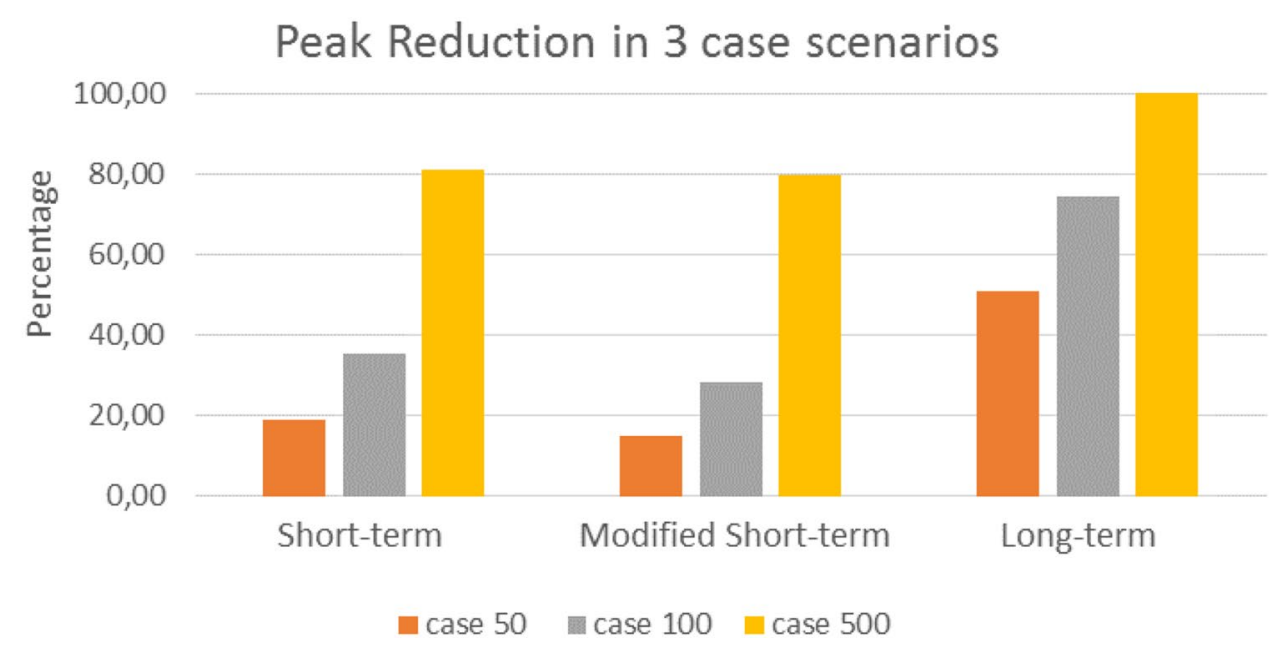

\begin{tabular}{|c|c|c|c|c|c|c|}
\hline \multirow[t]{3}{*}{ Case } & \multicolumn{6}{|c|}{ Average load factor (10 runs) } \\
\hline & \multicolumn{2}{|c|}{ Short-term } & \multicolumn{2}{|c|}{ Modified short-term } & \multicolumn{2}{|c|}{ Long-term } \\
\hline & Before & After & Before & After & Before & After \\
\hline 50 & 0.53 & 0.58 & 0.53 & 0.55 & 0.52 & 0.58 \\
\hline 100 & 0.53 & 0.60 & 0.53 & 0.57 & 0.52 & 0.60 \\
\hline 500 & 0.53 & 0.69 & 0.53 & 0.66 & 0.52 & 0.70 \\
\hline
\end{tabular}




\section{Conclusion}

This study analyzes the efficiency of implementing V2G technology in peak load management in public infrastructure. Three different scenarios representing the real parking plan in public infrastructures, such as an airport, were modeled. An algorithm for the implementation of a changing time window scheduling for charging and discharging of the EVs was designed. Using a typical load profile of such an infrastructure and predefined values for the peak and valley threshold, the algorithm was applied in all three different scenarios with different populations of EVs. The effectiveness of peak reduction using the V2G charge-discharge algorithm was analyzed and the results were compared in all the scenarios. From the results, it is concluded that the peak reduction is possible with the V2G implementation for the EVs in the parking place. The effectiveness of peak reduction depends essentially on the number of EVs in the parking place and the stay hours of the EVs. The results also show that for the scenarios such as long-term parking and short-term parking with fewer varying parking patterns, this V2G scheduling algorithm could be implemented and used to cope with the peak demands in the connected load systems. Even with a large number of electric vehicles in the future, V2G scheduling will bring advantages both for the EV user and for the grid.

Acknowledgements The authors acknowledge the fruitful discussion with their college Moritz Quandt regarding the simulation of test scenarios.

Authors' contributions This article is based on the master thesis of Harish Dhanasekaran done at BIBA - Bremer Institut für Produktion und Logistik $\mathrm{GmbH}$ and supervised by Prof. Michael Freitag from the University of Bremen. Juan Daniel Arango, Dimitri Denhof and AnnKathrin Rohde contributed during the development of the thesis as well as structuring and writing this manuscript. All authors edited and approved the final manuscript.

Funding This research is part of the project GEREGELT - "Integrated control for the energy self-sufficient operation of large-scale infrastructure based on environmental data", funded by the German Federal Ministry of Education and Research (BMBF) under Reference Number: 01LY1411A.

Availability of data and material The load profile used for this study was obtained from the modeling software energyPRO. After installing the program, the load profile can be found in the folder: $C: \backslash U s e r s \backslash$ user_namelDocumentslenergyPRO_Data\Deutsch \ahreszeitreihen nach Standardlastprofile \Strom $\backslash 2016$

\section{Compliance with ethical standards}

Conflict of interest On behalf of all authors, the corresponding author states that there is no conflict of interest.

\section{References}

1. Arbeitsgemeinschaft Energiebilanzen e.V.; EEFA; Statistisches Bundesamt (2016) Evaluation tables of the energy balance for Germany

2. Wu DW, Wang RZ (2006) Combined cooling, heating and power: a review. Prog Energy Combust Sci 32(5-6):459-495. https://doi. org/10.1016/j.pecs.2006.02.001

3. MacDougall W (2013) Electromobility in Germany: Vision 2020 and Beyond. Germany Trade and Invest

4. Zhou Y, Li X (2015) Vehicle to grid technology: a review, pp 9031-9036. https://doi.org/10.1109/chicc.2015.7261068

5. Yang Z, Yu S, Lou W et al (2011) $P^{2}$ : privacy-preserving communication and precise reward architecture for $\mathrm{V} 2 \mathrm{G}$ networks in smart grid. IEEE Trans Smart Grid 2(4):697-706. https://doi. org/10.1109/tsg.2011.2140343

6. Yuan X, Wang X, Zuo J (2013) Renewable energy in buildings in China-a review. Renew Sustain Energy Rev 24:1-8. https ://doi.org/10.1016/j.rser.2013.03.022

7. Paulus M, Borggrefe F (2011) The potential of demand-side management in energy-intensive industries for electricity markets in Germany. Appl Energy 88(2):432-441. https://doi. org/10.1016/j.apenergy.2010.03.017

8. EPEX Spot (2017) EPEX Spot Market data DE-LU. December 2017. https://www.epexspot.com/en/market-data/dayah eadauction/chart/auction-chart/2017-12-01/DE_LU/0d. Accessed 14 Feb 2018

9. Bundesverband der Energie und Wasserwirtschaft e.V. (2017) BDEW-Strompreisanalyse Mai 2017: Haushalt und Industrie, Berlin

10. Crespo Del Granado P, Pang Z, Wallace SW (2016) Synergy of smart grids and hybrid distributed generation on the value of energy storage. Appl Energy 170:476-488. https://doi. org/10.1016/j.apenergy.2016.01.095

11. Christensen L, Klauenberg J, Kveiborg O et al (2017) Suitability of commercial transport for a shift to electric mobility with Denmark and Germany as use cases. Res Transp Econ 64:4860. https://doi.org/10.1016/j.retrec.2017.08.004

12. Total number of electric cars in Germany 2008-2018, Statistic. https://www.statista.com/statistics/646075/total-numbe r-electric-cars-germany/. Accessed 08 Mar 2019

13. IEA - International Energy Agency (2017) Global EV Outlook 2017. IEA

14. Jóźwicka E/M (2016) Electric vehicles in Europe

15. Lacey G, Putrus G, Bentley E (2017) Smart EV charging schedules: supporting the grid and protecting battery life. IET Electr Syst Transp 7(1):84-91. https://doi.org/10.1049/ iet-est.2016.0032

16. Masoum AS, Deilami S, Moses PS et al (2011) Smart load management of plug-in electric vehicles in distribution and residential networks with charging stations for peak shaving and loss minimisation considering voltage regulation. IET Gener Trans Distrib 5(8):877. https://doi.org/10.1049/iet-gtd.2010.0574

17. Letendre SE, Kempton W (2002) The V2G concept: a new model for power? Public Utilities Fortnightly, pp 16-26

18. Loisel R, Pasaoglu G, Thiel C (2014) Large-scale deployment of electric vehicles in Germany by 2030: an analysis of grid-tovehicle and vehicle-to-grid concepts. Energy Policy 65:432-443. https://doi.org/10.1016/j.enpol.2013.10.029

19. EMD International $A / S$ (2018) EnergyPro. EMD International $A / S$, Denmark

20. Das Kraftfahrt-Bundesamt (2017) Kraftfahrt-Bundesamtes (KBA) Statistics. https://www.kba.de/DE/Statistik/Fahrzeuge/Neuzu lassungen/MonatlicheNeuzulassungen/monatl_neuzulassu ngen_node.html. Accessed 17 Dec 2017 
21. RWE Mobility (2017) RWE eStation Smart Product Specifications. https://www.rwe-mobility.com/web/cms/en/1231990/produ cts-services/emobility-produkte/rwe-estation-smart/. Accessed 18 Dec 2017

22. Barry Sole, Helena Selle, Ralf Oestreicher (eds) (2017) Market Place Based energy Management for PEV Grid Integration

23. Tomoko BLECH, Ryoko KUSUMI, Marta MARINELLI (ed) (2017) CHAdeMO V2X protocol: design concept, benefits and worldwide applications

24. Bishop JDK, Axon CJ, Bonilla D et al (2013) Evaluating the impact of V2G services on the degradation of batteries in PHEV and EV. Appl Energy 111:206-218. https://doi.org/10.1016/j.apene rgy.2013.04.094
25. Sharma DD, Singh SN (2014) Electrical load profile analysis and peak load assessment using clustering technique: IEEE PES general meeting conference and exposition, IEEE, National Harbor, $\mathrm{MD}$

Publisher's Note Springer Nature remains neutral with regard to jurisdictional claims in published maps and institutional affiliations. 